Proceedings of the 2007 Winter Simulation Conference

S. G. Henderson, B. Biller, M.-H. Hsieh, J. Shortle, J. D. Tew, and R. R. Barton, eds.

\title{
KERNEL ESTIMATION FOR QUANTILE SENSITIVITIES
}

\author{
Guangwu Liu \\ L. Jeff Hong \\ Department of Industrial Engineering and Logistics Management \\ The Hong Kong University of Science and Technology \\ Clear Water Bay, Hong Kong, CHINA
}

\begin{abstract}
Quantiles, also known as value-at-risk in financial applications, are important measures of random performance. Quantile sensitivities provide information on how changes in the input parameters affect the output quantiles. In this paper, we study the estimation of quantile sensitivities using simulation. We propose a new estimator by employing kernel method and show its consistency and asymptotic normality for i.i.d. data. Numerical results show that our estimator works well for the test problems.
\end{abstract}

\section{INTRODUCTION}

Quantiles are important measures of random performance. The $\alpha$-quantile of a continuous random variable $L$ is a value $q_{\alpha}$ such that $\operatorname{Pr}\left\{L \leq q_{\alpha}\right\}=\alpha$ for any pre-specified $\alpha$ value $(0<\alpha<1)$. It provides tail information of a distribution that is often missed by other widely used measures, e.g., mean and variance.

Quantiles have been adopted by many industries as major measures of random performance. In financial industry, the quantile, also known as the value-at-risk (VaR), is widely used as a measure of capital adequacy. For instance, banks and other large capitalization registrants are required to report their VaRs by various regulations. For a thorough introduction to $\mathrm{VaR}$ and its applications in financial industry, one can read the survey paper of Duffie and Pan (1997) or the monograph of Jorion (2001). In service industry, quantile is often used as a measure of service quality. For instance, the out-of-hospital systems often use the 0.9-quantile of the times taken to respond to emergency request and to transport patients to a hospital to measure the service quality (Austin and Schull 2003). Quantiles have also been used as billing measures in some circumstances. For instance, some Internet service providers (ISP) charge their users based on the 0.95 -quantile of the traffic load in a billing cycle (Goldenberg et al. 2004).
Quantile sensitivities provide information on how changes in the input parameters affect the output quantile performance. They play important roles in modeling, analysis and management of random performances. Since we typically use a model to model the random performance, and the model always has constants, controllable and uncontrollable parameters. For the constants in the model, the sensitivities with respect to them provide a measure of model robustness. If the sensitivities with respect to these constants are high, then the model is not robust to the changes in these constants. Since the constants are often used only for convenience, then modification of the model may be necessary. For the controllable parameters, sensitivities provide useful information on how to adjust the parameters to manage the quantile performance, and they can also be used to manage risk and to solve stochastic optimization problems. For the uncontrollable parameters, the sensitivities can be viewed as risk measures of the quantiles. If the sensitivities with respect to certain parameters are high, then small changes in these parameters may cause the output quantiles to change significantly. Then the risks with respect to these parameters are high.

Estimating sensitivities of expectations and long-run averages have been studied extensively in the simulation literature. Readers can refer to L'Ecuyer (1991) and Fu (2006) for comprehensive reviews. Typical methods include perturbation analysis, the likelihood ratio/score function method and the weak derivative method. The estimation of quantile sensitivities has only been studied very recently in Hong (2007). Hong (2007) shows that the quantile sensitivity can be written as a conditional expectation. Then he proposes a batching estimator based on the conditional expectation, and proves that the estimator is consistent and follows an asymptotic normal distribution.

Kernel method has been studied extensively in the area of nonparametric statistic and is applied to estimate conditional expectations (see, for instance, Bosq 1998). In this paper, we use kernel method to estimate the quantile 
sensitivities. We show that our estimator is consistent and asymptotically normally distributed for i.i.d. data.

The rest of the paper is organized as follows: Section 2 describes the problem mathematically and introduces our kernal estimator of the quantile sensitivity. The consistency and asymptotic normality of the estimator are studied in Sections 3. Section 4 discusses several implementation issues of the estimator. Numerical results are reported in Section 5, followed by the conclusions in Section 6 .

\section{BACKGROUND}

Suppose that the random performance can be modeled as $L(\theta)$, where $\theta \in \Theta$ is a parameter of the model and $\Theta$ is an open set in $\mathbb{R}$. Plenty of real-life applications can be modeled in this way. For instance, in queueing systems $\theta$ can be the arrival rate or service rate with $L(\theta)$ being the system time, while in financial models $\theta$ can represent market volatility, interest rate or some other market parameters with $L(\theta)$ being the price of some security under this parameter setting. The parameter $\theta$ can be multi-dimensional, but all through this paper we consider scalar $\theta$ only. This will not limit the applicability of our analysis, since every dimension can be considered individually for a multi-dimensional parameter.

Let $q_{\alpha}(\theta)$ be the $\alpha$-quantile of $L(\theta)$ for any $\theta \in \Theta$. In this paper we are interested in the sensitivity (derivative) of $q_{\alpha}(\theta)$ with respect to $\theta$, denoted by $q_{\alpha}^{\prime}(\theta)$.

We make the following assumptions.

Assumption 1. The pathwise derivative $L^{\prime}(\theta)$ exists w.p.1 for any $\theta \in \Theta$, and there exists a random variable $Y$ with $\mathrm{E}[Y]<\infty$, such that

$$
\left|L\left(\theta_{2}\right)-L\left(\theta_{1}\right)\right| \leq Y\left|\theta_{2}-\theta_{1}\right|
$$

for any $\theta_{1}, \theta_{2} \in \Theta$.

Assumption 2. For any $\theta \in \Theta, L(\theta)$ has a continuous density in a neighborhood of $q_{\alpha}(\theta)$, and $q_{\alpha}(\theta)$ is differentiable with respect to $\theta$.

Assumption 3. For any $\theta \in \Theta$, let

$$
l(t ; \theta)=\mathrm{E}\left[L^{\prime}(\theta) \mid L(\theta)=t\right]
$$

Then $l(t ; \theta)$ is continuous at $t=q_{\alpha}(\theta)$.

Then Hong and Liu (2007) prove the following lemma.

Lemma 1. If Assumptions 1-3 are satisfied, then

$$
q_{\alpha}^{\prime}(\theta)=\mathrm{E}\left[L^{\prime}(\theta) \mid L(\theta)=q_{\alpha}(\theta)\right]
$$

Remark 1. Hong (2007) also proves this lemma under more restrictive assumptions.
Based on the conditional-expectation form of Lemma 1 , this paper investigates the estimation of $q_{\alpha}^{\prime}(\theta)$ using simulation.

Let $D(\theta)=L^{\prime}(\theta)$. By Assumption 1, $D(\theta)$ exists w.p.1. Note that $D(\theta)$ is also a random variable. For simplicity we let $\left(L_{i}, D_{i}\right)$ denote $\left(L_{i}(\theta), D_{i}(\theta)\right)$ if there is no ambiguity. Suppose that we have i.i.d. data $\left\{\left(L_{i}, D_{i}\right), 1 \leq i \leq n\right\}$, where $L_{i}$ is an observation of the random performance while $D_{i}$ is its sample-path derivative with respect to $\theta$. In fact, for a large amount of systems the sample-path derivatives can be obtained with only little additional computation effort. For instance, for a market value of a firm at some pre-specified due date which follows log-normal distribution, the samplepath derivative with respect to the market volatility can be easily calculated based on the sample path itself. One is referred to Broadie and Glasserman (1996) for the method of deriving sample-path derivatives in many financial models. Another example is the queueing system. If the system times of customers in a $G I / G / 1$ queue are considered as random performance, then with little additional computation effort the sample-path gradients can be obtained using Infinitesimal Perturbation Analysis (IPA). For details of the application of IPA in queueing networks, one can refer to Glasserman (1991), and the references therein.

In this paper, we propose a kernel estimator to estimate $q_{\alpha}^{\prime}(\theta)$ using a set of i.i.d. data $\left\{\left(L_{i}, D_{i}\right), 1 \leq i \leq n\right\}$. Before introducing the estimator, we briefly review the kernel method.

By definition, a kernel on $\mathbb{R}$, denoted by $K$, is a bounded symmetric density such that $K(u) \rightarrow 0$ as $|u| \rightarrow \infty$ and $\int_{-\infty}^{\infty} u^{2} K(u) d u<\infty$ (Bosq 1998). For instance, the density of a normal random variable is a kernel. Kernel method has been extensively studied in the area of nonparametric statistics. Researchers have found that kernel method has several advantages: it is easy to compute and robust; and it reaches optimal rates in terms of the quadratic error. For more details on kernel estimation, one can refer to Bosq (1998). Typically, the kernel method involves a smoothing parameter $\delta_{n}$, which satisfies that, as $n \rightarrow \infty, \delta_{n} \rightarrow 0$ and $n \delta_{n} \rightarrow \infty$. All through the paper, we let $K$ denote the kernel and $q_{\alpha}$ denote $q_{\alpha}(\theta)$ if there is no ambiguity.

With the above preliminary knowledge, the kernel estimator that we propose for $q_{\alpha}^{\prime}(\theta)$ is expressed as follows (denoted by $V_{n}$ ):

$$
V_{n}=\frac{\sum_{i=1}^{n} D_{i} \cdot K\left(\frac{\hat{q}_{\alpha}^{n}-L_{i}}{\delta_{n}}\right)}{\sum_{i=1}^{n} K\left(\frac{\hat{q}_{\alpha}^{n}-L_{i}}{\delta_{n}}\right)},
$$

where $\hat{q}_{\alpha}^{n}$ is the $\alpha$ sample quantile of $\left\{L_{i}, 1 \leq i \leq n\right\}$, i.e., $\hat{q}_{\alpha}^{n}=L_{\lceil n \alpha\rceil: n}$ with $L_{i: n}$ being the $i$-th order statistic from the $n$ observations of $L$.

All through the paper we assume that $(L, D)$ is a continuous bivariate random variable with joint density $f(x, y)$. 
Also, for any non-negative integers $m$, we define

$$
g_{m}(x)=\int_{-\infty}^{\infty} t^{m} f(x, t) d t
$$

and

$$
h_{m}(x)=\int_{-\infty}^{\infty}|t|^{m} f(x, t) d t .
$$

Then by elementary calculus, Equation (1) can be re-written as

$$
q_{\alpha}^{\prime}(\theta)=\frac{g_{1}\left(q_{\alpha}\right)}{g_{0}\left(v_{\alpha}\right)}
$$

Remark 2. In general we assume that $(L, D)$ has joint density $f(x, y)$. As a special case, $(L, D)$ can be a degenerate bivariate random variable, i.e., $D=D(L)$ can be viewed as a function of L. Our method is also valid for this degenerate case.

The rest of the paper will focus on the consistency and asymptotic normality of the proposed estimator. Some implementation issues will also be discussed.

To make the paper easier to follow, in the rest of this section we state several preliminary results, which will used many times in our analysis afterwards.

Lemma 2 (Bachner's Lemma). Suppose that $K(y)$ is a Borel function satisfying the conditions: $\sup _{-\infty<y<\infty}|K(y)|<\infty$, $\int_{-\infty}^{+\infty}|K(y)| d y<\infty$ and $\lim _{y \rightarrow \infty}|y K(y)|=0$. Let $f(y)$ satisfy that $\int_{-\infty}^{+\infty}|f(y)| d y<\infty$ and let $\left\{b_{n}\right\}$ be a sequence of positive constants satisfying $\lim _{n \rightarrow \infty} b_{n}=0$. Let

$$
f_{n}(x)=\frac{1}{b_{n}} \int_{-\infty}^{+\infty} K\left(\frac{y}{b_{n}}\right) f(x-y) d y .
$$

For every $x$, if $f(\cdot)$ is continuous at $x$, then

$$
\lim _{n \rightarrow \infty} f_{n}(x)=f(x) \int_{-\infty}^{+\infty} K(y) d y .
$$

Proof of the Bachner's Lemma can be found in Parzen (1962), and a direct implication of it is as follows:

Lemma 3. For any non-negative integers $m$ and $l>0$. Suppose that $\int_{-\infty}^{\infty} K^{l}(u) d u<\infty$ and $\mathrm{E}\left[|D|^{m}\right]<\infty$. If $g_{m}(x)$ is continuous at $x=t$, then

$$
\mathrm{E}\left[\frac{1}{\delta_{n}} K^{l}\left(\frac{t-L}{\delta_{n}}\right) D^{m}\right] \rightarrow g_{m}(t) \int_{-\infty}^{\infty} K^{l}(u) d u
$$

If $h_{m}(x)$ is continuous at $x=t$, then

$$
\mathrm{E}\left[\frac{1}{\delta_{n}} K^{l}\left(\frac{t-L}{\delta_{n}}\right)|D|^{m}\right] \rightarrow h_{m}(t) \int_{-\infty}^{\infty} K^{l}(u) d u .
$$

\section{ASYMPTOTIC PROPERTIES OF THE KERNEL ESTIMATOR}

In the previous section we have reviewed the preliminary results and introduced a kernel estimator, $V_{n}$. We will focus on its asymptotic properties in this section. We show that it is consistent and follows asymptotic normality.

We first define some necessary notation. We define

$$
V_{n}(y)=\frac{\sum_{i=1}^{n} D_{i} \cdot K\left(\frac{y-L_{i}}{\delta_{n}}\right)}{\sum_{i=1}^{n} K\left(\frac{y-L_{i}}{\delta_{n}}\right)} .
$$

Moreover, we let $\stackrel{P}{\longrightarrow}$ and $\Rightarrow$ denote convergence in probability and convergence in distribution, respectively, and $f^{(m)}$ denote the $m$-th derivative of the function $f$.

\subsection{Consistency of $V_{n}$}

In this subsection we show that $V_{n}$ converges to $q_{\alpha}^{\prime}(\theta)$ in probability. The general idea of the proof is as follows: we first prove that $V_{n}\left(q_{\alpha}\right)$ converges to $q_{\alpha}^{\prime}(\theta)$ in probability; and then we show that $V_{n}-V_{n}\left(q_{\alpha}\right)$ converges to 0 in probability. Main results of this subsection are stated as follows:

Theorem 1. Suppose that $\mathrm{E}\left[D^{2}\right]<\infty$ and $n \delta_{n} \rightarrow \infty$. If $g_{0}(t)$, $g_{1}(t)$ and $g_{2}(t)$ are continuous at $t=q_{\alpha}$, and $g_{0}\left(q_{\alpha}\right)>0$, then

$$
V_{n}\left(q_{\alpha}\right) \stackrel{P}{\longrightarrow} q_{\alpha}^{\prime}(\theta)
$$

Theorem 1 shows that $V_{n}\left(q_{\alpha}\right)$ is a consistent estimator of $q_{\alpha}^{\prime}(\theta)$. However, it's not directly applicable since it requires knowing the $\alpha$-quantile, $q_{\alpha}$, which is typically unknown in practice. A natural way to circumvent this difficulty is to replace $q_{\alpha}$ by its estimator $\hat{q}_{\alpha}^{n}$, producing the estimator $V_{n}$. Before the analysis of consistency of $V_{n}$, we first make another assumption on the kernel $K$ and then establish a lemma.

Assumption 4. For $m=1,2,3,4,\left[K^{(m)}\right]^{2}$ is integrable and $y K^{(m)}(y) \rightarrow 0$ as $|y| \rightarrow \infty$.

Remark 3. This assumption on the kernel is easy to satisfy. For instance, a normal density fulfills all these requirements. 
Lemma 4. Suppose that Assumption 4 is satisfied. If $g_{2}(t)$ and $g_{1}^{(4)}(t)$ are continuous at $t=q_{\alpha}$, then for $m=1,2,3,4$,

$$
\mathrm{E}\left[\frac{1}{n \delta_{n}^{m+1}} \sum_{i=1}^{n} K^{(m)}\left(\frac{q_{\alpha}-L_{i}}{\delta_{n}}\right) D_{i}\right] \rightarrow g_{1}^{(m)}\left(v_{\alpha}\right),
$$

and

$$
\operatorname{Var}\left[\frac{1}{n \delta_{n}^{m+1}} \sum_{i=1}^{n} K^{(m)}\left(\frac{q_{\alpha}-L_{i}}{\delta_{n}}\right) D_{i}\right]=\frac{\sigma_{m}^{2}+o(1)}{n \delta_{n}^{2 m+1}},
$$

where

$$
\sigma_{m}^{2}=g_{2}\left(q_{\alpha}\right) \int_{-\infty}^{\infty}\left[K^{(m)}(t)\right]^{2} d t
$$

Now we are ready to state the main theorem of this subsection.

Theorem 2. Suppose that $\mathrm{E}\left[D^{2}\right]<\infty, g_{0}\left(q_{\alpha}\right)>0$, and that $n \delta_{n}^{2}$ converges to some positive constant or $\infty$. If $g_{2}(t)$, $g_{0}^{(4)}(t)$ and $g_{1}^{(4)}(t)$ are continuous at $t=q_{\alpha}$, then $V_{n}$ is a consistent estimator for $q_{\alpha}^{\prime}(\theta)$.

\subsection{Asymptotic Normality of $V_{n}$}

In this subsection we show that $V_{n}$ has an asymptotic normal distribution, which can be used to construct confidence intervals of $q_{\alpha}^{\prime}(\theta)$.

The basic ideas behind the proof is similar to the previous one. We first establish the asymptotic normality for $V_{n}\left(q_{\alpha}\right)$, with convergence rate $\sqrt{n \delta_{n}}$. Then we show that $\sqrt{n \delta_{n}}\left(V_{n}\left(\hat{q}_{\alpha}^{n}\right)-V_{n}\left(q_{\alpha}\right)\right)$ converges to 0 in probability. Then by Slutsky's Theorem we obtain the asymptotic normality of $V_{n}$. Main results are listed as follows.

Theorem 3. Suppose that $n \delta_{n} \rightarrow \infty$. For some $\gamma>0$, $\mathrm{E}\left[|D|^{2+\gamma}\right]<\infty$ and $h_{2+\gamma}(t)$ is continuous at $t=q_{\alpha}$. Also, $g_{2}(t), g_{1}^{(2)}(t)$ and $g_{0}^{(2)}(t)$ are continuous at $t=q_{\alpha}$. Then, if $n \delta_{n}^{5} \rightarrow c$ as $n \rightarrow \infty$,

$$
\sqrt{n \delta_{n}}\left[V_{n}\left(q_{\alpha}\right)-q_{\alpha}^{\prime}(\theta)\right] \Rightarrow \mu+\sigma N(0,1)
$$

and if $n \delta_{n}^{5} \rightarrow 0$ as $n \rightarrow \infty$,

$$
\sqrt{n \delta_{n}}\left[V_{n}\left(q_{\alpha}\right)-q_{\alpha}^{\prime}(\theta)\right] \Rightarrow \sigma N(0,1)
$$

where

$$
\mu=\frac{c}{g_{0}^{2}\left(q_{\alpha}\right)}\left[g_{0}\left(q_{\alpha}\right) g_{1}^{\prime \prime}\left(q_{\alpha}\right)-g_{1}\left(q_{\alpha}\right) g_{0}^{\prime \prime}\left(q_{\alpha}\right)\right] \int_{-\infty}^{\infty} t^{2} K(t) d t
$$

and

$$
\sigma^{2}=\frac{g_{0}\left(q_{\alpha}\right) g_{2}\left(q_{\alpha}\right)-g_{1}^{2}\left(q_{\alpha}\right)}{g_{0}^{3}\left(q_{\alpha}\right)} \int_{-\infty}^{\infty} K^{2}(t) d t
$$

Lemma 5. Suppose that $n \delta_{n}^{3}$ converges to some positive constant or $\infty$. If $g_{2}(t), g_{0}^{(4)}(t)$ and $g_{1}^{(4)}(t)$ are continuous at $t=q_{\alpha}$, then

$$
\sqrt{n \delta_{n}}\left(V_{n}-V_{n}\left(q_{\alpha}\right)\right) \stackrel{P}{\longrightarrow} 0 .
$$

Combining Theorem 3 and Lemma 5 together yields the following central limit theorem for $V_{n}$.

Theorem 4. Suppose that $n \delta_{n}^{3}$ converges to some positive constant or $\infty$. For some $\gamma>0, \mathrm{E}\left[|D|^{2+\gamma}\right]<\infty$ and $h_{2+\gamma}(t)$ is continuous at $t=q_{\alpha}$. Also, $g_{2}(t), g_{0}^{(4)}(t)$ and $g_{1}^{(4)}(t)$ are continuous at $t=q_{\alpha}$. Then, if $n \delta_{n}^{5} \rightarrow c$ as $n \rightarrow \infty$,

$$
\sqrt{n \delta_{n}}\left(V_{n}-q_{\alpha}^{\prime}(\theta)\right) \Rightarrow \mu+\sigma N(0,1)
$$

and if $n \delta_{n}^{5} \rightarrow 0$ as $n \rightarrow \infty$,

$$
\sqrt{n \delta_{n}}\left(V_{n}-q_{\alpha}^{\prime}(\theta)\right) \Rightarrow \sigma N(0,1)
$$

where $\mu$ and $\sigma$ are defined in Theorem 3 .

\section{IMPLEMENTATION ISSUES}

In the previous sections we have shown that the estimator we proposed is statistically efficient. However, in order to use it in practice, several implementation issues need to be considered. This section will discuss the selection of $\delta_{n}$ and how to construct confidence intervals.

\subsection{Selection of $\delta_{n}$}

We have obtained the asymptotic variance of $V_{n}$ in the previous analysis. In this subsection we will discuss the implementation issue on how to select $\delta_{n}$ for $V_{n}$, with the criterion being minimizing the asymptotic mean square error of $V_{n}$. Then the key of the problem becomes how to approximate the bias of $V_{n}$.

Direct analysis on bias of $V_{n}$ is difficult. But we note that difference between $V_{n}$ and $V_{n}\left(q_{\alpha}\right)$ is negligible in some sense. Hence the problem can be reduced to analyze bias of $V_{n}\left(q_{\alpha}\right)$. Let

Before the discussion we specify some notation first.

$$
R_{n}(y)=\frac{1}{n \delta_{n}} \sum_{i=1}^{n} D_{i} \cdot K\left(\frac{y-L_{i}}{\delta_{n}}\right)
$$




\section{Liu and Hong}

and

$$
Q_{n}(y)=\frac{1}{n \delta_{n}} \sum_{i=1}^{n} K\left(\frac{y-L_{i}}{\delta_{n}}\right)
$$

Then

$$
V_{n}(y)=\frac{R_{n}(y)}{Q_{n}(y)} .
$$

Similar to the analysis in Section 3, we can easily show that $R_{n}\left(\hat{q}_{\alpha}^{n}\right)$ and $Q_{n}\left(\hat{q}_{\alpha}^{n}\right)$ are consistent estimators of $g_{1}\left(q_{\alpha}\right)$ and $g_{0}\left(q_{\alpha}\right)$, respectively.

First, we note that

$$
\begin{aligned}
V_{n}- & V_{n}\left(q_{\alpha}\right) \\
= & \frac{1}{Q_{n}\left(\hat{q}_{\alpha}^{n}\right) Q_{n}\left(q_{\alpha}\right)}\left\{Q_{n}\left(q_{\alpha}\right)\left[R_{n}\left(\hat{q}_{\alpha}^{n}\right)-R_{n}\left(q_{\alpha}\right)\right]\right. \\
& \left.-R_{n}\left(q_{\alpha}\right)\left[Q_{n}\left(\hat{q}_{\alpha}^{n}\right)-Q_{n}\left(q_{\alpha}\right)\right]\right\} .
\end{aligned}
$$

We expand $R_{n}\left(\hat{q}_{\alpha}^{n}\right)-R_{n}\left(q_{\alpha}\right)$ using Young's Form of Taylor's Theorem (Serfling 1980) and assume that the higher order terms can be ignored. Then an approximation can be expressed as:

$R_{n}\left(\hat{q}_{\alpha}^{n}\right)-R_{n}\left(q_{\alpha}\right) \approx \frac{1}{n \delta_{n}^{2}} \sum_{i=1}^{n} D_{i} K^{\prime}\left(\frac{q_{\alpha}-L_{i}}{\delta_{n}}\right)\left(\hat{q}_{\alpha}^{n}-q_{\alpha}\right)$

By Lemma 4 we know that $\frac{1}{n \delta_{n}^{2}} \sum_{i=1}^{n} D_{i} K^{\prime}\left(\frac{q_{\alpha}-L_{i}}{\delta_{n}}\right)$ converges to $g_{1}^{\prime}\left(q_{\alpha}\right)$ in probability. Then it is reasonable to expect that $\mathrm{E}\left[R_{n}\left(\hat{q}_{\alpha}^{n}\right)-R_{n}\left(q_{\alpha}\right)\right]$ is of the same order as $\mathrm{E}\left[\hat{q}_{\alpha}^{n}-q_{\alpha}\right]$. It has been well known that under mild conditions, $\mathrm{E}\left[\hat{q}_{\alpha}^{n}-q_{\alpha}\right]$ is of order $1 / n$ (see, for instance, David 1981). Hence, $\mathrm{E}\left[R_{n}\left(\hat{q}_{\alpha}^{n}\right)-R_{n}\left(q_{\alpha}\right)\right]$ is of order $1 / n$.

Similar argument holds for $\mathrm{E}\left[Q_{n}\left(\hat{q}_{\alpha}^{n}\right)-Q_{n}\left(q_{\alpha}\right)\right]$ and hence it is reasonable for us to expect that $\mathrm{E}\left[V_{n}-V_{n}\left(q_{\alpha}\right)\right]$ is of order $1 / n$.

Furthermore, similar to the analysis in Hong and Liu (2007) we can show that under mild conditions, for large $n$,

$$
\mathrm{E}\left[V_{n}\left(q_{\alpha}\right)-q_{\alpha}^{\prime}(\theta)\right]=\mu_{q} \delta_{n}^{2}+o\left(\delta_{n}^{2}\right)
$$

where

$$
\mu_{q}=\frac{g_{1}^{\prime \prime}\left(q_{\alpha}\right)-q_{\alpha}^{\prime}(\theta) g_{0}^{\prime \prime}\left(q_{\alpha}\right)}{g_{0}\left(q_{\alpha}\right)} \int_{-\infty}^{\infty} t^{2} K(t) d t
$$

We assume that $n \delta_{n}^{2} \rightarrow \infty$. Then compared with $\mathrm{E}\left[V_{n}\left(q_{\alpha}\right)-\right.$ $\left.q_{\alpha}^{\prime}(\theta)\right], \mathrm{E}\left[V_{n}-V_{n}\left(q_{\alpha}\right)\right]$ is of smaller order and can be neglected. Therefore, an approximation for the mean square error of $V_{n}$ can be expressed as:

$$
\mathrm{E}\left[\left(V_{n}-q_{\alpha}^{\prime}(\theta)\right)^{2}\right] \approx \frac{\sigma^{2}}{n \delta_{n}}+\mu_{q}^{2} \delta_{n}^{4}
$$

Minimizing the above approximation of mean square error, we reach the optimal selection of $\delta_{n}$, denoted by $\delta_{n}^{*}$ :

$$
\delta_{n}^{*}=d_{q} \cdot n^{-\frac{1}{5}}
$$

where

$$
d_{q}=\left(\frac{\sigma^{2}}{4 \mu_{q}^{2}}\right)^{\frac{1}{5}}
$$

During implementation, one can conduct several simulation runs to estimate $d_{q}$.

Let

$$
G_{n}(y)=\frac{1}{n \delta_{n}} \sum_{i=1}^{n} D_{i}^{2} K\left(\frac{y-L_{i}}{\delta_{n}}\right)
$$

Then, similar to the analysis in Section 3 we can show that $G_{n}\left(\hat{q}_{\alpha}^{n}\right), R_{n}\left(\hat{q}_{\alpha}^{n}\right)$ and $Q_{n}\left(\hat{q}_{\alpha}^{n}\right)$ are consistent estimators of $g_{2}\left(q_{\alpha}\right), g_{1}\left(q_{\alpha}\right)$ and $g_{0}\left(q_{\alpha}\right)$, respectively.

Hence, the asymptotic variance $\sigma^{2}$ can be consistently estimated by

$$
\hat{S}_{n}=\int_{-\infty}^{\infty} K^{2}(t) d t\left[G_{n}\left(\hat{q}_{\alpha}^{n}\right) Q_{n}\left(\hat{q}_{\alpha}^{n}\right)-R_{n}^{2}\left(\hat{q}_{\alpha}^{n}\right)\right] /\left[Q_{n}^{3}\left(\hat{q}_{\alpha}^{n}\right)\right]
$$

Moreover, $g_{0}^{\prime \prime}\left(q_{\alpha}\right)$ and $g_{1}^{\prime \prime}\left(q_{\alpha}\right)$ can be estimated by the finite-difference approach. Let $s$ be the step size of finite difference (for instance, one can choose $s=20 \%\left|\hat{q}_{\alpha}^{n}\right|$ if $\left.\left|\hat{q}_{\alpha}^{n}\right|>0\right)$. Then we may estimate $g_{1}^{\prime \prime}\left(q_{\alpha}\right)$ and $g_{0}^{\prime \prime}\left(q_{\alpha}\right)$ by

$$
\hat{g}_{1}^{\prime \prime}=\frac{R_{n}\left(\hat{q}_{\alpha}^{n}+s\right)+R_{n}\left(\hat{q}_{\alpha}^{n}-s\right)-R_{n}\left(\hat{q}_{\alpha}^{n}\right)}{s^{2}}
$$

and

$$
\hat{g}_{0}^{\prime \prime}=\frac{Q_{n}\left(\hat{q}_{\alpha}^{n}+s\right)+Q_{n}\left(\hat{q}_{\alpha}^{n}-s\right)-Q_{n}\left(\hat{q}_{\alpha}^{n}\right)}{s^{2}},
$$

respectively.

Hence, $\mu_{q}$ can be consistently estimated by

$$
\hat{\mu}_{q}=\frac{\hat{g}_{1}^{\prime \prime}-V_{n} \hat{g}_{0}^{\prime \prime}}{Q_{n}\left(\hat{q}_{\alpha}^{n}\right)} \int_{-\infty}^{\infty} t^{2} K(t) d t
$$

Therefore, during implementation, we can conduct several simulation runs to determine $d_{q}$. Typically, we first 


\section{Liu and Hong}

choose $\delta_{n}$ to be $n^{-1 / 5}$ and estimate $d_{q}$ by

$$
\hat{d}_{q}=\left(\frac{\hat{S}_{n}}{4 \hat{\mu}_{q}^{2}}\right)^{\frac{1}{5}}
$$

Then we set the new $\delta_{n}$ to be $\hat{d}_{q} \cdot n^{-1 / 5}$ and estimate $d_{q}$ again. After several iterations we stop and obtain an estimate for $d_{q}$, which can be used in the main simulation study.

\subsection{Constructing Confidence Intervals}

In the discussion in the previous subsection, we know that a near optimal choice for $\delta_{n}$ is $d_{q} \cdot n^{-1 / 5}$ if the criterion is to minimize the mean square error of the estimator. However, if we are interested in constructing confidence intervals of $q_{\alpha}^{\prime}(\theta)$, then by Theorem 4 , we prefer that $n \delta_{n}^{5} \rightarrow 0$. Then $d_{q} \cdot n^{-1 / 5}$ violates this requirement. In this case, we suggest to let $\delta_{n}=d_{q} \cdot n^{-1 / 3}$. Then $V_{n}$ has a smaller bias but a larger variance. Then the confidence intervals have better coverage probabilities.

Specifically, an asymptotic valid $100(1-\beta) \%$ confidence interval of $q_{\alpha}^{\prime}(\theta)$ is

$$
\left(V_{n}-z_{1-\beta / 2} \hat{S}_{n} / \sqrt{n \delta_{n}}, V_{n}+z_{1-\beta / 2} \hat{S}_{n} / \sqrt{n \delta_{n}}\right)
$$

where $z_{1-\beta / 2}$ is the $1-\beta / 2$ quantile of the standard normal distribution.

\section{NUMERICAL STUDY}

In this section we will consider two numerical examples to illustrate the performance of our estimator. The first one is an investment problem in financial industry and the second one is the reliability problem in the service industry. In both examples we may derive the true values of the quantile sensitivities. Therefore, we can test the performance of our estimator by comparing it with the true values. For more complex systems, theoretical values may not be derived analytically. However, our estimator may still be applied.

In both of these examples, we let $K$ be the density of the standard normal random variable and consider $\alpha=0.9$.

\subsection{An Investment Problem}

A portfolio is composed of three risky assets. The annual rates of return of these assets are denoted by $X_{1}, X_{2}$ and $X_{3}$, and the percentages of the total fund allocated to the assets are denoted by $\theta=\left(\theta_{1}, \theta_{2}, \theta_{3}\right)^{\prime}$. Suppose that $X=$ $\left(X_{1}, X_{2}, X_{3}\right)^{\prime}$ follows a multivariate normal distribution with mean $\mu=(0.06,0.15,0.25)^{\prime}$ and the covariance matrix

$$
\Sigma=\left(\begin{array}{ccc}
0.0004 & -0.0006 & -0.0009 \\
-0.0006 & 0.1000 & 0.0044 \\
-0.0009 & 0.0044 & 0.0484
\end{array}\right)
$$

Then the portfolio annual rate of return is

$$
L(\theta, X)=\theta_{1} X_{1}+\theta_{2} X_{2}+\theta_{3} X_{3},
$$

it follows a normal distribution with mean $\theta^{\prime} \mu$ and variance $\theta^{\prime} \Sigma \theta$. Then the quantile sensitivities of $L(\theta, X)$ can be calculated analytically. Suppose that we are interested in the sensitivity of $q_{\alpha}(L(\theta, X))$, with respect to $\theta_{3}$, at the point $\theta=(0.2,0.3,0.5)^{\prime}$. By simple calculation, we know that

$$
\frac{\partial q_{\alpha}(L(\theta, X))}{\partial \theta_{3}}=0.25+0.2135 \cdot z_{\alpha}
$$

where $z_{\alpha}$ is the inverse function of the standard normal distribution function evaluated at $\alpha$.

On the other hand, since $\frac{\partial L(\theta, X)}{\partial \theta_{3}}=X_{3}$, the gradient for each sample path can be obtained. Then we can estimate $\partial q_{\alpha}(L(\theta, X)) / \partial \theta_{3}$ using the kernel estimator we proposed.

The estimated MSEs of the point estimator and the observed coverage probabilities of the $90 \%$ confidence interval with respect to different sample sizes are plot in Figures 1 and 2. The plots are based on 500 independent replications. From these figures, we see that the point estimator and the confidence interval have the desired properties.

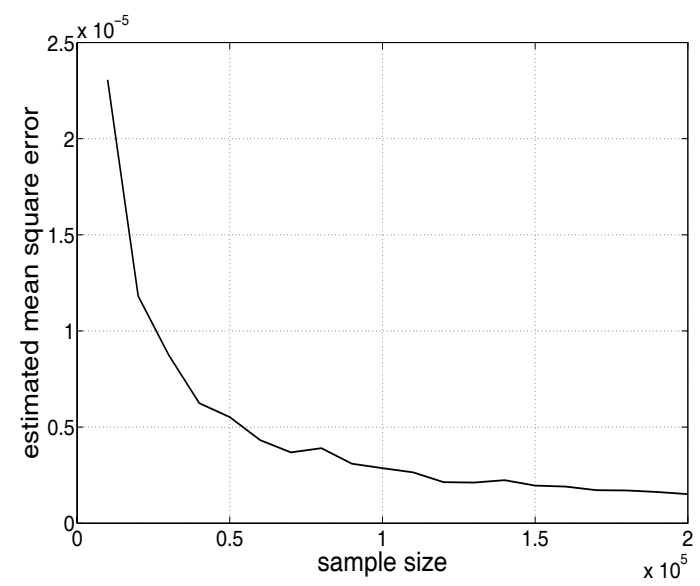

Figure 1: MSE of the quantile sensitivity estimator for the investment example.

\subsection{A Reliability Problem}

A system has three components in sequence. If one of the component fails, then the whole system fails. Suppose that the times to failure of the three components can be modeled as $X_{i} / \theta_{i}$ where $\theta_{i}$ is the failure rate and $X_{i}$ follows 


\section{Liu and Hong}

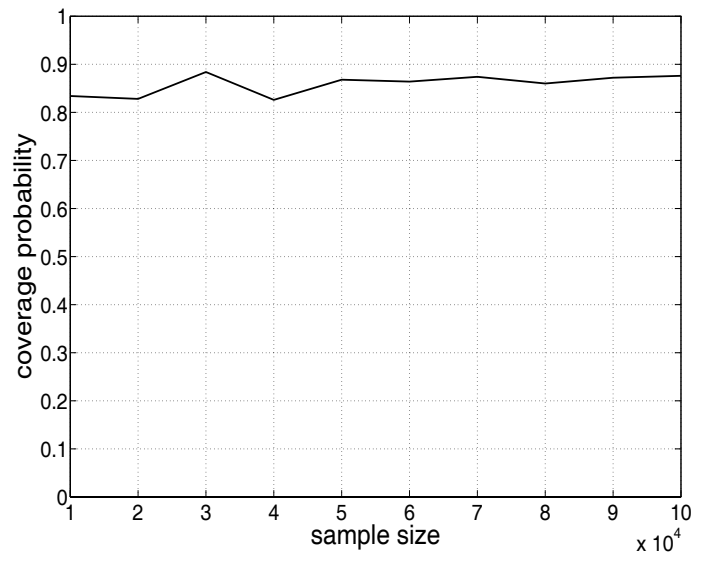

Figure 2: Coverage probability of $90 \%$ confidence interval for the investment example.

an exponential distribution with rate 1 for all $i=1,2,3$. Then the time to failure of the system is

$$
L(\theta, X)=\min \left\{\frac{X_{1}}{\theta_{1}}, \frac{X_{2}}{\theta_{2}}, \frac{X_{3}}{\theta_{3}}\right\} .
$$

It can be seen that $L(\theta, X)$ follows exponential distribution with rate $\theta_{1}+\theta_{2}+\theta_{3}$. In this example we are interested in the sensitivity of $q_{\alpha}(L(\theta, X))$ with respect to $\theta_{1}$, at $\theta=(1,1,1)$. By simple calculation, we know that

$$
\frac{\partial q_{\alpha}(L(\theta, X))}{\partial \theta_{1}}=\frac{\log (1-\alpha)}{\left(\theta_{1}+\theta_{2}+\theta_{3}\right)^{2}} .
$$

On the other hand, the sample path derivative of $L(\theta, X)$ w.r.t $\theta_{1}$ is

$$
\frac{\partial L(\theta, X)}{\partial \theta_{1}}= \begin{cases}-\frac{X_{i}}{\theta_{i}^{2}} & \text { if } \frac{X_{i}}{\theta_{i}}=\min \left\{\frac{X_{1}}{\theta_{1}}, \frac{X_{2}}{\theta_{2}}, \frac{X_{3}}{\theta_{3}}\right\}, \\ 0 & \text { otherwise. }\end{cases}
$$

Then we can estimate the sensitivity of $q_{\alpha}(L(\theta, X))$ using the method we proposed.

The estimated MSEs of the point estimator and the observed coverage probabilities of the $90 \%$ confidence interval with respect to different sample sizes are plot in Figures 3 and 4 . The plots are based on 500 independent replications. From these figures, we see that the point estimator and the confidence interval have the desired properties.

\section{CONCLUSIONS}

In this paper we propose a kernel estimator for estimating quantile sensitivities. For independent and identically distributed data, we show the consistency and normality of the estimator. Numerical study shows that our estimator works well for the testing examples.

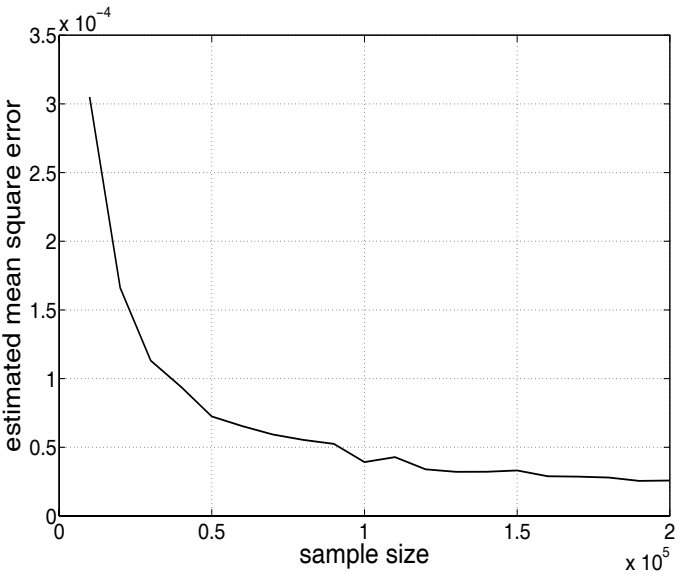

Figure 3: MSE of the quantile sensitivity estimator for the reliability example.

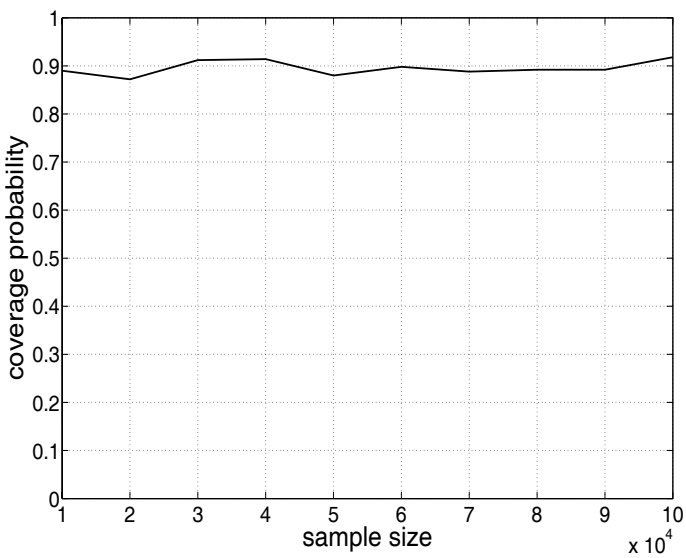

Figure 4: Coverage probability of $90 \%$ confidence interval for the reliability example.

\section{ACKNOWLEDGMENTS}

This research was partially supported by Hong Kong Research Grants Council grant CERG 613706.

\section{REFERENCES}

Austin, P. and M. Schull. 2003. Quantile regression: A statistical tool for out-of-patient research. Academic Emergency Medicine, 10 789-797.

Bosq, D. 1998. Nonparametric statistics for stochastic processes. Springer, New York.

Broadie, M. and P. Glasserman. 1996. Estimating security price derivatives using simulation. Management Science, 42 (2): 269-285.

David, H. A. 1981. Order Statistics. Second Edition. John Wiley \& Sons, New York.

Duffie, D. and J. Pan. 2001. An overview of value at risk. Journal of Derivatives, 4:7-49. 
Fu, M. C. 2006. Gradient estimation. S. G. Henderson and B. L. Nelson, ed. Handbooks in Operations Research and Management Science: Simulation, Elsevier.

Glasserman, P. 1991. Gradient estimation via perturbation analysis. Kluwer Academic Publishers, Boston.

Glynn, P. W. and D. L. Iglehart. 1985. Large-sample theory for standardized time series: an overview. Proceedings of the 1985 Winter Simulation Conference. D. Gantz, G. Blais, S. Solomon (eds).

Goldenberg, D. K., L. Qiu, H. Xie, Y. R.. Yang, and Y. Zhang. 2004. Optimizing cost and performance of multihoming. Proc. SIGCOMM 2004,79-92.

Hong, L. J. 2007. Estimating quantile sensitivities. Working paper, Department of Industrial Engineering and Logistics Management, The Hong Kong University of Science and Technology, Hong Kong, China.

Hong, L. J. and G. Liu. 2007. Estimating probability sensitivities through terminating or steady-state simulations. Working paper, Department of Industrial Engineering and Logistics Management, The Hong Kong University of Science and Technology, Hong Kong, China.

Jorion, P. 2001. Value at Risk, Second Edition. Wiley, New York, NY.

L'Ecuyer. 1991. An overview of derivative estimation. Proceedings of the 1991 Winter Simulation Conference: 207-217

Lehmann. E. L. 1999. Elements of large-sample theory. Springer, New York.

Parzen, E. 1962. On estimation of a probability density function and mode. The Annals of Mathematical Statistics, 33 (3): 1065-1076.

Serfling, R. J. 1980. Approximation theorems of mathematical statistics, John Wiley \& Sons, New York.

\section{AUTHOR BIOGRAPHIES}

GUANGWU LIU is a Ph.D. student in the Department of Industrial Engineering and Logistics Management at The Hong Kong University of Science and Technology. $\mathrm{He}$ is currently working on estimating sensitivities of risk measures. His e-mail address is <liugweust.hk>.

L. JEFF HONG is an assistant professor in industrial engineering and logistics management at The Hong Kong University of Science and Technology. His research interests include Monte-Carlo method, sensitivity analysis and simulation optimization. He is currently associate editors of Naval Research Logistics and ACM Transactions on Modeling and Computer Simulation. His e-mail address is <hongl@ust.hk>. 